\title{
Simplicial volume of Hilbert modular varieties
}

\author{
Clara Löh and Roman Sauer
}

\begin{abstract}
The simplicial volume introduced by Gromov provides a topologically accessible lower bound for the minimal volume. Lafont and Schmidt proved that the simplicial volume of closed, locally symmetric spaces of non-compact type is positive. In this paper, we extend this positivity result to certain non-compact locally symmetric spaces of finite volume, including Hilbert modular varieties. The key idea is to reduce the problem to the compact case by first relating the simplicial volume of these manifolds to the Lipschitz simplicial volume and then taking advantage of a proportionality principle for the Lipschitz simplicial volume. Moreover, using computations of Bucher-Karlsson for the simplicial volume of products of closed surfaces, we obtain the exact value of the simplicial volume of Hilbert modular surfaces.
\end{abstract}

Mathematics Subject Classification (2000). Primary 53C23; Secondary 53C35

Keywords. Simplicial volume, Hilbert modular varieties.

\section{Introduction and statement of results}

1.1. Simplicial volume. The simplicial volume of a manifold was introduced by Gromov [Gro82]: The simplicial volume of an $n$-dimensional, compact, oriented manifold $M$ (possibly with non-empty boundary $\partial M$ ) is defined by

$$
\|M, \partial M\|=\inf \left\{|c|_{1} ; c \in C_{n}(M) \text { relative fundamental cycle of }(M, \partial M)\right\} .
$$

Here $|c|_{1}$ denotes the $\ell^{1}$-norm of a chain in the singular chain complex $C_{n}(M)$ with real coefficients with respect to the basis of all singular simplices. Similarly, one defines the simplicial volume $\|M\|^{\text {lf }}$ for an open, oriented $n$-manifold $M$ by fundamental cycles in the locally finite chain complex $C_{n}^{\text {lf }}(M)$ with $\mathbb{R}$-coefficients:

$$
\|M\|^{\text {lf }}=\inf \left\{|c|_{1} ; c \in C_{n}^{\text {lf }}(M) \text { fundamental cycle of } M\right\} .
$$

In the sequel we suppress the superscript "lf" in the notation and just write $\|M\|$. Because both simplicial volumes are multiplicative with respect to finite coverings, it makes sense to define the simplicial volume of a non-orientable manifold $M$ as $\|\bar{M}\| / 2$ where $\bar{M}$ is the orientation double covering of $M$. Notice that the simplicial volume is invariant under proper homotopy equivalences. 
1.2. The volume estimate. One of the main motivations to introduce the simplicial volume is to establish a lower bound for the minimal volume by a homotopy invariant (proper homotopy invariant in the non-compact case). Gromov defined the minimal volume minvol $(M)$ of a smooth manifold $M$ as the infimum of volumes $\operatorname{vol}(M, g)$ over all complete Riemannian metrics $g$ on $M$ with sectional curvature pinched between -1 and 1 . The following fundamental inequality holds [Gro82, p. 12, p. 73] for all smooth $n$-manifolds $M$ :

$$
\|M\| \leq(n-1)^{n} n ! \cdot \operatorname{minvol}(M) .
$$

A basic question is whether $\operatorname{minvol}(M)>0$, and thus whether $\|M\|>0$. For example, for negatively curved locally symmetric spaces of finite volume one knows that the minimal volume is achieved by the locally symmetric metric [BCG95], [BCS05], [Sto06]. Gromov first proved that the minimal volume of closed locally symmetric spaces is positive. Connell and Farb gave a much more detailed proof of this result in [CF03]. They also proved that locally symmetric spaces of finite volume that do not have a local $\mathbb{H}^{2}$ - or $\operatorname{SL}(3, \mathbb{R}) / \mathrm{SO}(3, \mathbb{R})$-factor (thereby excluding Hilbert modular varieties) have a positive minimal volume within the Lipschitz class of the locally symmetric metric. ${ }^{1}$

Thurston proved that if $M$ has a complete Riemannian metric of finite volume with sectional curvature between $-k$ and -1 then $\|M\|>0$ [Gro82, Section 0.2]. Moreover, if $M$ is a compact locally symmetric space of non-compact type, then $\|M\|>0$ by a result of Lafont and Schmidt [LS06].

1.3. Lipschitz simplicial volume. The aim of this paper is to give explicit computations and estimates of the simplicial volume of certain locally symmetric spaces with $\mathbb{R}$-rank at least 2, namely, of Hilbert modular varieties.

However, the simplicial volume of non-compact manifolds is much less well behaved than the one for compact manifolds. For instance, both the proportionality principle [Gro82, Section 0.4] and the product formula [Gro82, Section 0.2] fail in general. Even worse, the simplicial volume of $\Gamma \backslash X$, where $X$ is a symmetric space and $\Gamma$ an arithmetic lattice of $\mathbb{Q}$-rank at least 3, vanishes [LöhS09].

The following metric variant of the simplicial volume, which was first considered by Gromov [Gro82] and is studied in detail in our paper [LöhS09], shows a much more decent behaviour.

Definition 1.1. Let $M$ be an oriented, $n$-dimensional Riemannian manifold without boundary. For a locally finite chain $c=\sum_{i \geq 0} a_{\sigma_{i}} \sigma_{i}$ define $\operatorname{Lip}(c) \in[0, \infty]$ as the supremum of the Lipschitz constants of the singular simplices $\sigma_{i}: \Delta^{n} \rightarrow M$

\footnotetext{
${ }^{1}$ Benson Farb informed us that positivity of the minimal volume in the finite volume case is erroneously stated in the cited reference without this Lipschitz constraint. However, Chris Connell explained to us how to modify their proof to get positivity of the minimal volume.
} 
where $\Delta^{n}$ carries the standard Euclidean metric. Then the Lipschitz simplicial volume $\|M\|_{\text {Lip }}$ of $M$ is defined as

$$
\|M\|_{\text {Lip }}=\inf \left\{|c|_{1} ; c \in C_{n}^{\text {lf }}(M) \text { fundamental cycle of } M \text { with } \operatorname{Lip}(c)<\infty\right\} .
$$

Note that $\|M\|_{\text {Lip }}=\|M\|$ for all closed Riemannian manifolds $M$ because singular homology and smooth singular homology are isometrically isomorphic with respect to the $\ell^{1}$-semi-norm [Löh06, Proposition 5.3].

The main advantage of the Lipschitz simplicial volume over the ordinary one is the presence of the following proportionality principles.

Theorem 1.2 (Gromov's proportionality principle, open-closed). Let $M$ be a closed Riemannian manifold, and let $N$ be a complete Riemannian manifold of finite volume. Assume the universal covers of $M$ and $N$ are isometric. Then

$$
\frac{\|M\|_{\text {Lip }}}{\operatorname{vol}(M)}=\frac{\|N\|_{\text {Lip }}}{\operatorname{vol}(N)}
$$

Proof. Gromov defines different notions of Lipschitz volumes [Gro82, Section 4.5], denoted by $\|V: \operatorname{vol}\|_{+}$and $\|V: \operatorname{vol}\|_{-}$, which also make sense for Riemannian manifolds $V$ with infinite volume. For a complete Riemannian manifold $V$ of finite volume he states the inequality [Gro82, third set of examples in Section 4.5]

$$
\|V: \operatorname{vol}\|_{-} \leq \frac{\|V\|}{\operatorname{vol}(V)} \leq\|V: \operatorname{vol}\|_{+} .
$$

The hypothesis of being stable at infinity [Gro82, proportionality theorem of Section 4.5] is always satisfied for complete Riemannian manifolds of finite volume (see the Example in loc. cit.), and thus the theorem in loc. cit. says that

$$
\|N: \operatorname{vol}\|_{-}=\|N: \operatorname{vol}\|_{+}=\frac{\|M\|}{\operatorname{vol}(M)} .
$$

Therefore, the inequality above yields the assertion.

Since the proof of Gromov's theorem is not very detailed, we refer the reader also to the following result [LöhS09], which one might use alternatively in Strategy 1.4 below.

Theorem 1.3 (Proportionality principle, non-positive curvature). Let $M$ and $N$ be complete, non-positively curved Riemannian manifolds of finite volume whose universal covers are isometric. Then

$$
\frac{\|M\|_{\text {Lip }}}{\operatorname{vol}(M)}=\frac{\|N\|_{\text {Lip }}}{\operatorname{vol}(N)}
$$


Our strategy to compute the simplicial volume for a locally symmetric space $M$ of non-compact type and finite volume consists of the following steps:

\section{Strategy 1.4.}

- Show that $\|M\|=\|M\|_{\text {Lip }}$ under certain conditions.

- By a theorem of Borel [Bor63], there is a compact locally symmetric space $N$ of non-compact type with the same universal covering as $M$; furthermore, both $M$ and $N$ are non-positively curved (see [Ebe96, Sections 2.1 and 2.2]).

- Use the positivity result of Lafont and Schmidt [LS06] to conclude $\|N\|>0$, or use other available specific computations of $\|N\|$.

- Apply Theorem 1.2 or 1.3 and the equality of the first step to get positivity or an explicit computation of $\|M\|$ from the one of $\|N\|$. Notice that both proportionality principles are applicable in view of the second step.

1.4. Relating simplicial volume and Lipschitz simplicial volume. The following general result helps to verify the first step in the strategy above for certain examples. It is proved in Section 2.

Theorem 1.5. Let $(M, d)$ be an open, Riemannian manifold. Assume that there is a compact submanifold $W \subset M$ with boundary $\partial W=\coprod_{i=1}^{m} W_{i}$ and connected components $W_{i}$ satisfying the following conditions:

(a) The complement $M-W$ and the disjoint union $\bigsqcup_{i=1}^{m} W_{i} \times(0, \infty)$ of cylinders are homeomorphic.

(b) Each $W_{i}$ has a finite cover $\bar{W}_{i} \rightarrow W_{i}$ that has a self-map $f_{i}: \bar{W}_{i} \rightarrow \bar{W}_{i}$ with $\operatorname{deg} f_{i} \notin\{-1,0,1\}$ with the following property: Let $f_{i}^{(k)}$ denote the $k$-fold composition of $f_{i}$. There is $C>0$ such that for every $k \geq 1$ the map

$$
F_{i}^{(k)}: \bar{W}_{i} \times[0,1] \rightarrow \bar{W}_{i} \times[k, k+1], \quad(x, t) \mapsto\left(f_{i}^{(k)}(x), t+k\right)
$$

has Lipschitz constant at most $C$ with respect to the metric on $\bar{W}_{i} \times(0, \infty)$ induced by $d$.

(c) Each $W_{i}$ has amenable fundamental group.

Then

$$
\|W, \partial W\|=\|M\|=\|M\|_{\text {Lip }} .
$$


1.5. Simplicial volume of locally symmetric spaces of finite volume. Next we present examples of locally symmetric spaces that satisfy the hypothesis of the previous theorem. In the sequel we focus on the case of higher $\mathbb{R}$-rank but we remark that it is not hard to verify the hypothesis for hyperbolic manifolds of finite volume or, more generally, locally symmetric spaces of $\mathbb{R}$-rank 1 . In particular, using Theorem 1.2 or 1.3, one obtains a new proof of Thurston's proportionality principle for hyperbolic manifolds [Gro82, Section 0.4].

Let us first fix the following notation.

Setup 1.6. Let $X$ be a connected symmetric space of non-compact type and let $G=\operatorname{Isom}(X)^{\circ}$ be the connected component of the identity in the isometry group of $X$. Then $G$ is a connected semi-simple Lie group with trivial center and no compact factors [Ebe96, Proposition 2.1.1 on p. 69]. Let $\boldsymbol{G}$ be a connected, semi-simple, linear algebraic group with trivial center such that $G=\boldsymbol{G}(\mathbb{R})$ (there always exists such $\boldsymbol{G}$ ). Assume that the $\mathbb{Q}$-rank of $\boldsymbol{G}(\mathbb{Q})$ is 1 , i.e., any maximal $\mathbb{Q}$-split torus of $\boldsymbol{G}(\mathbb{Q})$ is one-dimensional. Let $\Gamma \subset \boldsymbol{G}(\mathbb{Q})$ be a torsion-free, arithmetic lattice. By definition, one says that $\Gamma$ is a $\mathbb{Q}$-rank 1 lattice.

Section 3 gives a summary of the relevant notions about locally symmetric spaces. The following Theorem is proved in Section 4.

Theorem 1.7. We retain the assumptions of Setup 1.6. Assume that $\Gamma$ in addition satisfies the following conditions:

(a) The lattice $\Gamma$ is neat in the sense of Borel [Bor69, §17], and

(b) the connected components of the boundary of the Borel-Serre compactification of $M=\Gamma \backslash X$ have amenable fundamental groups.

Then $M$ satisfies the assumption of Theorem 1.5.

Applying the Strategy 1.4, we obtain the following positivity result:

Corollary 1.8. Under the hypotheses of the previous theorem we have $\|\Gamma \backslash X\|>0$.

1.6. Simplicial volume of Hilbert modular varieties. Consider a totally real number field $F$ of degree $d$ over $\mathbb{Q}$, that is, $F$ admits no complex embedding. Let $\mathcal{O}_{F}$ be its ring of integers. Let $\left\{\sigma_{1}, \ldots, \sigma_{d}\right\}$ be the set of all embeddings $\mathcal{O}_{F} \rightarrow \mathbb{R}$.

Then $\operatorname{SL}\left(2, \mathcal{O}_{F}\right)$ is a $\mathbb{Q}$-rank 1 lattice in $\operatorname{SL}(2, \mathbb{R})^{d}$ via the embedding

$$
A \mapsto\left(\sigma_{1}(A), \ldots, \sigma_{d}(A)\right) .
$$

In particular, $\operatorname{SL}\left(2, \mathcal{O}_{F}\right)$ acts on the $d$-fold product $\mathbb{H}^{2} \times \cdots \times \mathbb{H}^{2}$ of hyperbolic planes. 
If $\Gamma \subset \operatorname{SL}\left(2, \mathcal{O}_{F}\right)$ is a torsion-free, finite index subgroup then we call the quotient $\Gamma \backslash\left(\mathbb{H}^{2}\right)^{d}$ a Hilbert modular variety. In case $F$ is real quadratic, i.e., $F=\mathbb{Q}(\sqrt{a})$ for a square-free positive integer $a$, we call $\Gamma \backslash \mathbb{H}^{2} \times \mathbb{H}^{2}$ a Hilbert modular surface.

If $\Gamma$ is neat then $\Gamma \backslash\left(\mathbb{H}^{2}\right)^{d}$ satisfies the assumption of Theorem 1.7 [BJ06b, III.2.7]. Further, every torsion-free lattice has a neat subgroup of finite index [Bor69, §17]. Thus, by Corollary 1.8 and the volume estimate in Section 1.2 we obtain:

Corollary 1.9. Hilbert modular varieties have positive simplicial and positive minimal volume.

To our knowledge, this is the first class of examples of non-compact locally symmetric spaces of $\mathbb{R}$-rank at least 2 for which positivity of the simplicial or the minimal volume is known.

Bucher-Karlsson proved [BK07] that if the Riemannian universal covering of a closed Riemannian manifold $N$ is isometric to $\mathbb{H}^{2} \times \mathbb{H}^{2}$, then $\|N\|=\frac{3}{2 \pi^{2}} \operatorname{vol}(N)$. Thus, applying Strategy 1.4, yields following explicit computation.

Corollary 1.10. Let $\Sigma$ be a Hilbert modular surface. Then

$$
\|\Sigma\|=\frac{3}{2 \pi^{2}} \operatorname{vol}(\Sigma)
$$

Acknowledgments. The first author would like to thank the Graduiertenkolleg "Analytische Topologie und Metageometrie" at the WWU Münster for its financial support. The second named author would like to thank Uri Bader for numerous discussions about Lie groups and symmetric spaces, which helped a lot in the preparation of this paper. The second named author acknowledges support of the German Science Foundation (DFG), made through grant SA 1661/1-1.

\section{Proof of Theorem 1.5}

Proof of Theorem 1.5. We first show that $\|W, \partial W\| \leq\|M\| \leq\|M\|_{\text {Lip }}$ : By definition, $\|M\| \leq\|M\|_{\text {Lip. }}$. On the other hand, restricting a locally finite fundamental cycle $\tilde{c}$ of $M$ to the submanifold $W$, and pushing the resulting finite chain down to $W$ via the obvious homotopy equivalence $M \rightarrow W$ gives rise to a relative fundamental cycle $c$ of $(W, \partial W)$; by construction, $|c|_{1} \leq|\widetilde{c}|_{1}$. Therefore, $\|W, \partial W\| \leq\|M\|$.

It remains to prove the estimate $\|W, \partial W\| \geq\|M\|_{\text {Lip }}$ : Let $c \in C_{n}(W)$ be a relative fundamental cycle of $(W, \partial W)$. We now proceed in several steps: First, we use the given finite coverings and self-maps on the boundary components to extend $c$ to a locally finite chain $\tilde{c}$ on $M$. In the second step, we show that $\tilde{c}$ is a fundamental 
cycle of $M$. By refining the construction in the third step, we can build $\tilde{c}$ in such a way that is has small $\ell^{1}$-norm. In the last step, we show that $|\widetilde{c}|_{1}$ is indeed small enough, by applying Gromov's equivalence theorem.

Construction of a locally finite fundamental cycle $\tilde{c}$ of $M$ from $c$. In the following, we write $n=\operatorname{dim} M$. For $i \in\{1, \ldots, m\}$ let $p_{i}: \bar{W}_{i} \rightarrow W_{i}$ denote the given finite covering. Let $d_{i} \notin\{0,-1,1\}$ be the degree of the self-map $f_{i}: \bar{W}_{i} \rightarrow \bar{W}_{i}$. The chain

$$
z_{i}=\left.(\partial c)\right|_{W_{i}} \in C_{n-1}\left(W_{i}\right)
$$

is a fundamental cycle of $W_{i}$ and $\partial c=\sum_{i} z_{i}$. Let $\bar{z}_{i} \in C_{n-1}\left(\bar{W}_{i}\right)$ be the image of $z_{i}$ under the transfer homomorphism $C_{*}\left(W_{i}\right) \rightarrow C_{*}\left(\bar{W}_{i}\right)$ corresponding to $p_{i}$. Then $\bar{z}_{i}$ is a cycle representing $1 /\left|\operatorname{deg} p_{i}\right|$ times the fundamental class of $\bar{W}_{i}$ and satisfying $\left|\bar{z}_{i}\right|_{1} \leq\left|z_{i}\right|_{1}$ as well as $C_{n-1}\left(p_{i}\right)\left(\bar{z}_{i}\right)=z_{i}$. Because $f_{i}$ has degree $d_{i}$, we can find a chain $\bar{b}_{i} \in C_{n}\left(\bar{W}_{i}\right)$ such that

$$
\partial \bar{b}_{i}=1 / d_{i} \cdot C_{n-1}\left(f_{i}\right)\left(\bar{z}_{i}\right)-\bar{z}_{i} .
$$

In the third step of the proof, we will specify a suitable choice of $\bar{b}_{i}$.

There is a chain $\bar{u}_{i} \in C_{n}\left(\bar{W}_{i} \times[0,1]\right)$ with

$$
\partial \bar{u}_{i}=j_{1}\left(\bar{z}_{i}\right)-j_{0}\left(\bar{z}_{i}\right) \quad \text { and } \quad\left|\bar{u}_{i}\right|_{1} \leq n \cdot\left|\bar{z}_{i}\right|_{1},
$$

where $j_{k}: C_{*}\left(\bar{W}_{i}\right) \rightarrow C_{*}\left(\bar{W}_{i} \times[0, \infty)\right)$ denotes the map induced by the inclusion $\bar{W}_{i} \hookrightarrow \bar{W}_{i} \times\{k\} \hookrightarrow \bar{W}_{i} \times[0, \infty)$. For example, such a $\bar{u}_{i}$ can be found by triangulating the prism $\Delta^{n-1} \times[0,1]$ into $n$-simplices. Using the abbreviations

$$
\begin{aligned}
& \bar{w}_{i}=\bar{u}_{i}+j_{1}\left(\bar{b}_{i}\right) \in C_{n}\left(\bar{W}_{i} \times[0,1]\right), \\
& \bar{c}_{i}=\sum_{k \geq 0} \frac{1}{d_{i}^{k}} \cdot C_{n}\left(F_{i}^{(k)}\right)\left(\bar{w}_{i}\right) \in C_{n}^{\mathrm{lf}}\left(\bar{W}_{i} \times[0, \infty)\right),
\end{aligned}
$$

we define the (locally finite) chain

$$
\tilde{c}=c+\sum_{i=1}^{m} C_{n}\left(p_{i}\right)\left(\bar{c}_{i}\right) \in C_{n}^{\mathrm{lf}}(M) .
$$

Taking advantage of a smoothing operator [Löh06, Proposition 5.3] from the singular chain complex to the subcomplex generated by all smooth simplices, shows that we can assume without loss of generality that all the simplices occurring in the chains $\bar{w}_{i}$ and $c$ are smooth, and hence Lipschitz; in particular, all simplices in $\tilde{c}$ are Lipschitz. 
Properties of the chain $\tilde{c}$. The chain $\tilde{c}$ is a cycle because

$$
\begin{aligned}
\partial \bar{c}_{i} & =\sum_{k \geq 0} \frac{1}{d_{i}^{k}} \cdot C_{n-1}\left(F_{i}^{(k)}\right)\left(\partial \bar{w}_{i}\right) \\
& =\sum_{k \geq 0} \frac{1}{d_{i}^{k}} \cdot C_{n-1}\left(F_{i}^{(k)}\right)\left(j_{1}\left(\bar{z}_{i}\right)-j_{0}\left(\bar{z}_{i}\right)+j_{1}\left(\partial \bar{b}_{i}\right)\right) \\
& =\sum_{k \geq 0} \frac{1}{d_{i}^{k}} \cdot C_{n-1}\left(F_{i}^{(k)}\right)\left(\frac{1}{d_{i}} \cdot j_{1} \circ C_{n-1}\left(f_{i}\right)\left(\bar{z}_{i}\right)-j_{0}\left(\bar{z}_{i}\right)\right) \\
& =\sum_{k \geq 0} \frac{1}{d_{i}^{k+1}} \cdot j_{k+1} \circ C_{n-1}\left(f_{i}^{(k+1)}\right)\left(\bar{z}_{i}\right)-\sum_{k \geq 0} \frac{1}{d_{i}^{k}} \cdot j_{k} \circ C_{n-1}\left(f_{i}^{(k)}\right)\left(\bar{z}_{i}\right) \\
& =-\bar{z}_{i} .
\end{aligned}
$$

For every $x$ in the interior of $W$, the chains $c$ and $\tilde{c}$ give rise to the same class in $H_{n}(M, M-\{x\})$. Therefore, the cycle $\tilde{c}$ is a fundamental cycle of $M$. Moreover, $\operatorname{Lip}(\widetilde{c})<\infty$ follows directly from the uniform Lipschitz hypothesis for $\left(F_{i}^{(k)}\right)_{k \in \mathbb{N}}$.

Uniform boundary condition from amenability. Because $\pi_{1}\left(W_{i}\right)$ is amenable, the finite index subgroup $\pi_{1}\left(\bar{W}_{i}\right)$ is also amenable. Thus, the chain complex $C_{*}\left(\bar{W}_{i}\right)$ satisfies the uniform boundary condition of Matsumoto and Morita [MM85, Theorem 2.8] in degree $n-1$ (in fact, in every degree), i.e., there is a constant $K>0$ such that for any null-homologous cycle $\bar{z} \in C_{n-1}\left(\bar{W}_{i}\right)$ there is a chain $\bar{b} \in C_{n}\left(\bar{W}_{i}\right)$ with $\partial \bar{b}=\bar{z}$ and $|\bar{b}|_{1} \leq K \cdot|\bar{z}|_{1}$. By taking the maximum we may assume that we have the same constant $K$ for all $i \in\{1, \ldots, m\}$. Therefore, we can choose the $\bar{b}_{i}$ above in such a way that

$$
\left|\bar{b}_{i}\right|_{1} \leq K \cdot\left|1 / d_{i} \cdot C_{n-1}\left(f_{i}\right)\left(\bar{z}_{i}\right)-\bar{z}_{i}\right|_{1} \leq 2 K \cdot\left|\bar{z}_{i}\right|_{1},
$$

and again - by smoothing $\bar{b}_{i}$ - we may assume that all simplices occurring in $\bar{b}_{i}$ are smooth. This yields

$$
\begin{aligned}
|\widetilde{c}|_{1} & \leq|c|_{1}+\sum_{i=1}^{m}\left|\bar{c}_{i}\right|_{1} \\
& \leq|c|_{1}+\sum_{i=1}^{m} \sum_{k \geq 0} \frac{1}{d_{i}^{k}} \cdot\left(\left|\bar{u}_{i}\right|_{1}+\left|j_{1}\left(\bar{b}_{i}\right)\right|_{1}\right) \\
& \leq|c|_{1}+\sum_{i=1}^{m} \sum_{k \geq 0} \frac{2 K+n}{d_{i}^{k}} \cdot\left|\bar{z}_{i}\right|_{1} \\
& \leq|c|_{1}+2 m(2 K+n) n \cdot|\partial c|_{1} .
\end{aligned}
$$


Applying Gromov's equivalence theorem. From the discussion above we obtain that $\|M\|_{\text {Lip }} \leq \inf \left\{|c|_{1}+2 m(2 K+n) n \cdot|\partial c|_{1} ; c \in C_{n}(W)\right.$ relative fundamental cycle $\}$.

Then the amenability of each $\pi_{1}\left(W_{i}\right)$ and Gromov's equivalence theorem [Gro82, p. 57] imply that the right hand side is equal to $\|W, \partial W\|$.

\section{Review of cusp decomposition in $\mathbb{Q}$-rank 1}

We refer to the Setup 1.6. Our background reference is the book of Borel and Ji [BJ06b]. As in this book, we stick to the following notation:

Convention. An algebraic group defined over $\mathbb{Q}$ or $\mathbb{R}$ is denoted by a bold italic capital and its group of real points by the corresponding (ordinary) italic letter. Parabolic subgroups are always assumed to be proper and usually denoted by $\boldsymbol{P}$.

3.1. The Borel-Serre compactification - simply connected case. In the $\mathbb{Q}$-rank one case, the Borel-Serre compactification $\bar{X}$ of the symmetric space $X$ (the one of the locally symmetric space $\Gamma \backslash X$ will then be $\Gamma \backslash \bar{X}$, cf. Section 3.4) is of the form

$$
\bar{X}=X \cup \coprod_{\boldsymbol{P}} e(\boldsymbol{P}),
$$

where $\boldsymbol{P}$ runs over all rational parabolic subgroups of $\boldsymbol{G}$, and the boundary component $e(\boldsymbol{P})$ is a principal bundle over a symmetric space $X_{\boldsymbol{P}}$ with the unipotent radical $N_{\boldsymbol{P}}$ of $P$ as fiber. If one fixes a basepoint $x_{0} \in X$ and thus an identification $X=G / K$ (with $K=G_{x_{0}}$ maximal compact), then one can describe $e(\boldsymbol{P})$ as follows [BJ06b, Section III.9]: Let $P=N_{\boldsymbol{P}} A_{\boldsymbol{P}} M_{\boldsymbol{P}}$ be the rational Langlands decomposition of $P$ with respect to $x_{0}$ : Here $A_{\boldsymbol{P}}$ is a stable lift of the identity component of the real locus of the maximal $\mathbb{Q}$-split torus in the Levi-quotient $\boldsymbol{N}_{\boldsymbol{P}} \backslash \boldsymbol{P}$, and $M_{\boldsymbol{P}}$ is a stable lift of the real locus of the complement of this torus in $N_{\boldsymbol{P}} \backslash \boldsymbol{P}$. Write $K_{\boldsymbol{P}}=K \cap M_{\boldsymbol{P}}$ and $\Gamma_{P}=\Gamma \cap P$ and $\Gamma_{N_{P}}=\Gamma \cap N_{\boldsymbol{P}}$. Then $X_{\boldsymbol{P}}=M_{\boldsymbol{P}} / K_{\boldsymbol{P}}$ and $e(\boldsymbol{P})=N_{\boldsymbol{P}} \times X_{\boldsymbol{P}}$. The map [BJ06b, Section III.1, p. 272-275]

$$
\mu_{x_{0}}: N_{\boldsymbol{P}} \times A_{\boldsymbol{P}} \times X_{\boldsymbol{P}} \stackrel{\cong}{\rightarrow} X, \quad(n, a, m K) \mapsto n a m \cdot x_{0}
$$

is a diffeomorphism. If $\mu_{x_{0}}(n, a, z)=x$ then one calls $(n, a, z)$ the horospherical coordinates of $x \in X$. 
3.2. The rational root space decomposition. Let $g$ be the Lie algebra of $G$. Let $\mathfrak{a}_{\boldsymbol{P}}$ and $\mathfrak{n}_{\boldsymbol{P}}$ be the Lie algebra of $A_{\boldsymbol{P}}$ and $N_{\boldsymbol{P}}$, respectively. Associated with $\left(\mathfrak{g}, \mathfrak{a}_{\boldsymbol{P}}\right)$ is the system $\Phi\left(\mathfrak{g}, \mathfrak{a}_{\boldsymbol{P}}\right)$ of $\mathbb{Q}$-roots. For $\alpha \in \Phi\left(\mathfrak{g}, \boldsymbol{a}_{\boldsymbol{P}}\right)$ we write

$$
\mathfrak{g}_{\alpha}=\left\{Z \in \mathrm{g} ; \operatorname{ad}(H)(Z)=\alpha(H) Z \text { for all } H \in \boldsymbol{a}_{\boldsymbol{P}}\right\} .
$$

Furthermore, if $\Phi^{+}\left(\mathfrak{g}, \mathfrak{a}_{\boldsymbol{P}}\right)$ denotes the subset of positive roots, then we have

$$
\mathfrak{n}_{\boldsymbol{P}}=\bigoplus_{\alpha \in \Phi^{+}\left(\mathrm{g}, \mathrm{a}_{\boldsymbol{P}}\right)} \mathfrak{g}_{\alpha}
$$

Because $\boldsymbol{G}(\mathbb{Q})$ has $\mathbb{Q}$-rank 1 there is only one positive simple root $\alpha_{0} \in \Phi^{+}\left(\mathfrak{g}, \mathfrak{a}_{\boldsymbol{P}}\right)$, which defines a group isomorphism

$$
A_{\boldsymbol{P}} \stackrel{\cong}{\rightarrow} \mathbb{R}_{>0}^{*}, \quad a \mapsto a^{\alpha_{0}}:=\exp \circ \alpha_{0}(\log (a)) .
$$

We remark that the root decomposition above gives a grading of $\mathfrak{n}_{\boldsymbol{P}}$, and the adjoint action of $a \in A_{\boldsymbol{P}}$ on $\mathfrak{n}_{\boldsymbol{P}}$ is given by multiplication with $\lambda^{k}$ on $\mathfrak{g}_{k \alpha_{0}}$, where $\lambda=a^{\alpha_{0}}$.

3.3. The ends, metrically. For any $t \geq 0$ let $A_{\boldsymbol{P}, t}=\left\{a \in A_{\boldsymbol{P}} ; \alpha_{0}(\log a) \geq t\right\}$. Then

$$
V_{\boldsymbol{P}}(t)=\mu_{x_{0}}\left(N_{\boldsymbol{P}} \times A_{\boldsymbol{P}, t} \times X_{\boldsymbol{P}}\right)
$$

together with $e(\boldsymbol{P})$ defines a neighborhood of $e(\boldsymbol{P})$ for any $t \geq 0$. Next we describe the metric on $V_{\boldsymbol{P}}(t)$ in horospherical coordinates [BJ06b, Lemma III.20.7 on p. 402]: The tangent spaces of the three submanifolds $N_{\boldsymbol{P}} \times\{a\} \times\{z\}$ and $\{n\} \times A_{\boldsymbol{P}, t} \times\{z\}$ and $\{n\} \times\{a\} \times X_{\boldsymbol{P}}$ at every point $(n, a, z)$ are orthogonal. Let $d x^{2}, d a^{2}$, and $d z^{2}$ be the invariant metrics on $X, A_{\boldsymbol{P}}$, and $X_{\boldsymbol{P}}$, respectively, induced from the Killing form. Then we have

$$
d x^{2}=\sum_{a \in \Phi^{+}\left(\mathfrak{g}, \boldsymbol{a}_{\boldsymbol{P}}\right)} e^{-2 \alpha(\log (a))} h^{\alpha}(z) \oplus d a^{2} \oplus d z^{2},
$$

where $h^{\alpha}(z)$ is some metric on $g_{\alpha}$ that smoothly depends on $z$ but is independent of $a$.

3.4. The Borel-Serre compactification - locally symmetric case. Let $S$ denote a set of representatives of $\Gamma$-conjugacy classes of rational parabolic subgroups. There is a free $\Gamma$-action on $\bar{X}$ extending that of $X$, and the quotient $\Gamma \backslash \bar{X}$ is the Borel-Serre compactification of the locally symmetric space $\Gamma \backslash X$. The $\Gamma$-stabilizer of $e(\boldsymbol{P})$, and also of $V_{\boldsymbol{P}}(t)$ for $t \gg 1$, is $\Gamma_{\boldsymbol{P}}$, and two points in $V_{\boldsymbol{P}}(t)$ lie in the same $\Gamma$-orbit if and only if they lie in the same $\Gamma_{P}$-orbit. It turns out that

$$
\Gamma \backslash \bar{X}=\Gamma \backslash X \cup \coprod_{\boldsymbol{P} \in S} \Gamma_{P} \backslash e(\boldsymbol{P}) .
$$


Moreover, for $t \gg 1$ we have (in horospherical coordinates with respect to a basepoint)

$$
\Gamma_{\boldsymbol{P}} \backslash V_{\boldsymbol{P}}(t)=\Gamma_{\boldsymbol{P}} \backslash\left(N_{\boldsymbol{P}} \times A_{\boldsymbol{P}, t} \times X_{\boldsymbol{P}}\right)=\Gamma_{\boldsymbol{P}} \backslash\left(N_{\boldsymbol{P}} \times X_{\boldsymbol{P}}\right) \times A_{\boldsymbol{P}, t} .
$$

There is $t_{0}>0$ such that for all $t>t_{0}$ the subsets $\left(\Gamma_{P} \backslash V_{\boldsymbol{P}}(t)\right)_{\boldsymbol{P} \in S}$, of $\Gamma \backslash X$ are disjoint and the complement $\Gamma \backslash X-\bigcup_{\boldsymbol{P} \in S} \Gamma_{P} \backslash V_{\boldsymbol{P}}(t)$ is a compact submanifold with boundary. For $t>t_{0}$ we say that $\Gamma_{P} \backslash V_{\boldsymbol{P}}(t)$ is a cusp. The union of all cusps together with the boundary of $\Gamma \backslash \bar{X}$ is a collar neighborhood of the same boundary [JZ01, Propositions 2.3 and 2.4].

3.5. The boundary of the Borel-Serre compactification and neatness. Denote the image of $\Gamma_{P}$ under the projection $P=N_{\boldsymbol{P}} A_{\boldsymbol{P}} M_{\boldsymbol{P}} \rightarrow M_{\boldsymbol{P}}$ by $\Gamma_{M_{\boldsymbol{P}}}$. We assume that $\Gamma$ is neat in the sense of Borel [Bor69, §17]. By definition, $\Gamma$ is neat if the multiplicative subgroup of $\mathbb{C}^{\times}$generated by the eigenvalues of $f(\gamma) \in \operatorname{GL}(n, \mathbb{C})$ is torsion-free for any $\gamma \in \Gamma$ and any $\mathbb{Q}$-embedding $f: G \rightarrow \operatorname{GL}(n, \mathbb{C})$. Every lattice has a neat subgroup of finite index, and a neat lattice is also torsion-free [Bor69, §17]. Then $\Gamma_{M_{P}} \subset M_{P}$ is a torsion-free, uniform lattice in $M_{\boldsymbol{P}}$, and one obtains a short exact sequence

$$
0 \rightarrow \Gamma_{N_{P}} \rightarrow \Gamma_{P} \rightarrow \Gamma_{M_{P}} \rightarrow 0 .
$$

Moreover, $\Gamma_{\boldsymbol{P}} \backslash\left(N_{\boldsymbol{P}} \times X_{\boldsymbol{P}}\right)$ is a fiber bundle over the locally symmetric space $B_{\boldsymbol{P}}=$ $\Gamma_{M_{\boldsymbol{P}}} \backslash X_{\boldsymbol{P}}$ whose fiber is the nil-manifold $\Gamma_{N_{\boldsymbol{P}}} \backslash N_{\boldsymbol{P}}$. The assumption that $\Gamma$ is neat is needed to ensure that $B_{\boldsymbol{P}}$ is a manifold. The homeomorphism $\alpha_{0} \circ \log : A_{\boldsymbol{P}, t} \rightarrow \mathbb{R}_{>t}$ identifies the metric on $A_{\boldsymbol{P}, t}$ with the standard Euclidean metric on $\mathbb{R}$. Every cusp of $\Gamma \backslash X$ is topologically a cylinder $\Gamma_{P} \backslash\left(N_{\boldsymbol{P}} \times X_{\boldsymbol{P}}\right) \times \mathbb{R}_{>t}$. From the discussion in Section 3.3 we see that in the locally symmetric metric the nil-manifold fiber in $B_{\boldsymbol{P}}$ shrinks exponentially while the base stays fixed when the $A_{\boldsymbol{P}, t}$-component goes to infinity.

\section{Proof of Theorem 1.7}

Proof of Theorem 1.7. We know from the discussions in Section 3 (especially 3.4), to the notation of which we freely refer, that the locally symmetric space $M=\Gamma \backslash X$ has finitely many cusps $\left(\Gamma_{\boldsymbol{P}} \backslash V_{\boldsymbol{P}}(t)\right)_{\boldsymbol{P} \in S}$, where $S$ is a set of representatives of $\Gamma$-conjugacy classes of rational parabolic subgroups and $t>0$ is sufficiently large. The complement $W$ of $M$ minus the union of cusps is diffeomorphic to the Borel-Serre compactification of $M$. It remains to verify hypothesis (b) of Theorem 1.5 for every cusp $\Gamma_{P} \backslash V_{\boldsymbol{P}}(t)$. We consider the rational Langlands decomposition $P=N_{\boldsymbol{P}} A_{\boldsymbol{P}} M_{\boldsymbol{P}}$ with respect to a basepoint $x_{0} \in X$ such that $A_{\boldsymbol{P}}$ and $M_{\boldsymbol{P}}$ are defined over $\mathbb{Q}$. This is always possible [BJ06a, Proposition 2.2]. Then $\Lambda_{\boldsymbol{P}}:=\Gamma_{N_{\boldsymbol{P}}}\left(\Gamma \cap M_{\boldsymbol{P}}\right)$ is a finite 
index subgroup of $\Gamma_{P}$ [BJ06a, Remark 2.7]. In horospherical coordinates, the cusp is given by

$$
\Gamma_{\boldsymbol{P}} \backslash V_{\boldsymbol{P}}(t)=\Gamma_{\boldsymbol{P}} \backslash\left(N_{\boldsymbol{P}} \times X_{\boldsymbol{P}}\right) \times A_{\boldsymbol{P}, t} .
$$

Moreover, $\bar{W}_{\boldsymbol{P}}=\Lambda_{\boldsymbol{P}} \backslash\left(N_{\boldsymbol{P}} \times X_{\boldsymbol{P}}\right)$ is a finite cover of $W_{\boldsymbol{P}}=\Gamma_{\boldsymbol{P}} \backslash\left(N_{\boldsymbol{P}} \times X_{\boldsymbol{P}}\right)$.

Note for the following that $N_{\boldsymbol{P}} \times A_{\boldsymbol{P}} \times X_{\boldsymbol{P}}$ inherits a $G$-action from $X$ via $\mu_{x_{0}}$. In coordinates, multiplication by $a_{0} \in A_{\boldsymbol{P}}$ is given by $a_{0}(n, a, x)=\left(c_{a_{0}}(n), a_{0} a, x\right)$ where $c_{a_{0}}(n)=a_{0} n a_{0}^{-1}$ [BJ06a, Section 2]. Remember also that the (positive) root system $\Phi^{+}\left(\mathfrak{g}, \mathfrak{a}_{\boldsymbol{P}}\right)$ in Section 3.2 consists of positive, integral multiples of the single, positive simple root $\alpha_{0}$.

The next lemma provides the non-trivial self-maps (even self-coverings) assumed in hypothesis (b).

Lemma 4.1. There is a non-trivial self-covering $f_{\boldsymbol{P}}$ of $\Lambda_{\boldsymbol{P}} \backslash\left(N_{\boldsymbol{P}} \times X_{\boldsymbol{P}}\right)$ such that the following square commutes:

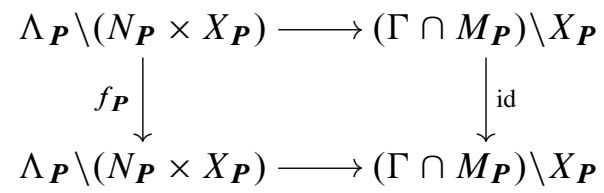

Moreover, the lift of $f_{\boldsymbol{P}}$ to the universal cover is given by $(n, x) \mapsto\left(a_{0} n a_{0}^{-1}, x\right)$ for some $a_{0} \in A_{\boldsymbol{P}}$ with $\lambda=a_{0}^{\alpha_{0}}>1$.

Proof. Because $A_{\boldsymbol{P}}$ commutes with $\Gamma \cap M_{\boldsymbol{P}}$, the map $c_{a_{0}} \times \mathrm{id}_{X_{\boldsymbol{P}}}$ for some $a_{0} \in A_{\boldsymbol{P}}$ induces a non-trivial self-covering on the $\Lambda_{\boldsymbol{P}}$-quotient provided $c_{a_{0}}\left(\Gamma_{N_{\boldsymbol{P}}}\right)$ is a proper, finite index subgroup of $\Gamma_{N_{\boldsymbol{P}}}$. The latter condition can be checked on the level of Lie algebras. Because $N_{\boldsymbol{P}}$ and $A_{\boldsymbol{P}}$ are defined over $\mathbb{Q}$, the Lie algebra $n_{\boldsymbol{P}}$ has a preferred rational structure and the decomposition of $\mathfrak{n}_{\boldsymbol{P}}$ in Section 3.2 is defined over $\mathbb{Q}$. Recall that the adjoint action of $a_{0}$ on $\mathfrak{n}_{\boldsymbol{P}}$ is given by the homomorphism $\varphi_{\lambda}: \mathfrak{n}_{\boldsymbol{P}} \rightarrow \mathfrak{n}_{\boldsymbol{P}}$ where $\lambda=a_{0}^{\alpha_{0}}$ and $\varphi_{\lambda}(n)=\lambda^{i} n$ for $n \in \mathfrak{g}_{i \alpha_{0}}$. The group $\Gamma_{N_{\boldsymbol{P}}}$ corresponds to a $\mathbb{Z}$-lattice in $\mathfrak{n}_{\boldsymbol{P}}$ by the exponential map. Dekimpe and Lee show that there is an integer $\lambda>1$ such that $\varphi_{\lambda}$ preserves this lattice [DL03, Lemma 5.2]. The corresponding $a_{0} \in A_{\boldsymbol{P}}$ with $a_{0}^{\alpha_{0}}=\lambda$ is the desired element.

Continuation of the proof of Theorem 1.7. Let $f_{\boldsymbol{P}}$ and $a_{0} \in A_{\boldsymbol{P}}$ and $\lambda>1$ be as in the previous lemma. Recall that $\Phi^{+}\left(\mathrm{g}, \boldsymbol{a}_{\boldsymbol{P}}\right)=\left\{\alpha_{0}, 2 \alpha_{0}, \ldots\right\}$, where $\alpha_{0}$ is the single positive simple root. Next we show that left multiplication by $a_{0}$ is Lipschitz with Lipschitz constant smaller than 1: First note that left multiplication by $a_{0}$ on the $A_{\boldsymbol{P}, t^{-}}$and $X_{\boldsymbol{P}}$-component of $V_{\boldsymbol{P}}(t)$ is an isometry or the identity, respectively. On the $N_{\boldsymbol{P}}$-component it is just the conjugation $c_{a_{0}}$. Let $\|V\|_{(n, a, z)}$ denote the norm of a 
tangent vector $V$ of $V_{\boldsymbol{P}}(t)$ at the point $(n, a, z)$. For $m \in \mathbb{N}$ and $N \in \mathfrak{g}_{m \alpha_{0}}$ we have according to Section 3.3

$$
\begin{aligned}
\left\|d c_{a_{0}}(N)\right\|_{\left(c_{a_{0}}(n), a a_{0}, z\right)} & =\left\|\lambda^{m} N\right\|_{\left(c_{a_{0}}(n), a a_{0}, z\right)} \\
& =\lambda^{-2 m} \lambda^{m}\|N\|_{(n, a, z)}<\|N\|_{(n, a, z)} .
\end{aligned}
$$

In particular, for any $k \in \mathbb{N}$, left multiplication by $a_{0}^{k}$ has a Lipschitz constant smaller than 1. Let $t_{0}=\alpha_{0}\left(\log a_{0}\right)>0$. If we identify $A_{\boldsymbol{P}, t}$ with $\mathbb{R}_{>t}$ by $\alpha_{0} \circ \log$, then the map on $\Lambda_{P} \backslash V_{\boldsymbol{P}}(t)$ induced by left multiplication by $a_{0}$ is just

$$
\Lambda_{\boldsymbol{P}} \backslash\left(N_{\boldsymbol{P}} \times X_{\boldsymbol{P}}\right) \times \mathbb{R}_{>t} \ni\left((n, z), t^{\prime}\right) \mapsto\left(f_{\boldsymbol{P}}(n, z), t_{0}+t^{\prime}\right) .
$$

Thus, after a suitable reparametrization of $\mathbb{R}_{>t}$, multiplication by $a_{0}^{k}$ is the desired map $F_{i}^{(k)}(i$ corresponds to $\boldsymbol{P}$ ) in hypothesis (b) of Theorem 1.5, which finishes the proof.

\section{References}

[BCG95] Gérard Besson, Gilles Courtois and Sylvestre Gallot, Entropies et rigidités des espaces localement symétriques de courbure strictement négative. Geom. Funct. Anal. 5 (5) (1995), 731-799. Zbl 0851.53032 MR 1354289

[BCS05] Jeffrey Boland, Chris Connell and Juan Souto, Volume rigidity for finite volume manifolds. Amer. J. Math. 127 (3) (2005), 535-550. Zbl 1078.53022 MR 2141643

[Bor63] Armand Borel, Compact clifford-klein forms of symmetric spaces. Topology 2 (1963), 111-122. Zbl 0116.38603 MR 0146301

[Bor69] Armand Borel, Introduction aux groupes arithmétiques. Publications de l'Institut de Mathématique de l'Université de Strasbourg, XV, Actualités Scientifiques et Industrielles 1341. Hermann, Paris 1996. Zbl 0186.33202 MR 0244260

[BJ06a] Armand Borel and Lizhen Ji, Compactifications of locally symmetric spaces. $J$. Differential Geom. 73 (2) (2006), 263-317. Zbl 1122.22005 MR 2226955

[BJ06b] Armand Borel and Lizhen Ji, Compactifications of symmetric and locally symmetric spaces. Math. Theory Appl., Birkhäuser, Boston 2006. Zbl 1100.22001 MR 2189882

[BK07] Michelle Bucher-Karlsson, The simplicial volume of closed manifolds covered by $\mathbb{H}^{2} \times \mathbb{H}^{2}$. J. Topol. 1 (3) (2008), 584-602. Zbl 05322831 MR 2417444

[CF03] Christopher Connell and Benson Farb, The degree theorem in higher rank. J. Differential Geom. 65 (1) (2003), 19-59. Zbl 1067.53032 MR 2057530

[DL03] Karel Dekimpe and Kyung Bai Lee, Expanding maps on infra-nilmanifolds of homogeneous type. Trans. Amer. Math. Soc. 355 (3) (2003) 1067-1077. Zbl 1009.37018 MR 2057530

[Ebe96] Patrick B. Eberlein, Geometry of nonpositively curved manifolds. Chicago Lectures in Mathematics. University of Chicago Press, Chicago 1996. Zbl 0883.53003 MR 1441541 
[Gro82] Michael Gromov, Volume and bounded cohomology. Inst. Hautes Études Sci. Publ. Math. 56 (1982), 5-99. Zbl 0516.53046 MR 0686042

[JZ01] Lizhen Ji and Maciej Zworski, Scattering matrices and scattering geodesics of locally symmetric spaces. Ann. Sci. École Norm. Sup. (4) 34 (3) (2001), 441-469. Zbl 1026.53026 MR 1949358

[LS06] Jean-François Lafont and Benjamin Schmidt, Simplicial volume of closed locally symmetric spaces of non-compact type. Acta Math. 197 (1) (2006), 129-143. Zbl 1111.57020 MR 2285319

[Löh06] Clara Löh, Measure homology and singular homology are isometrically isomorphic. Math. Z. 253 (1) (2006), 197-218. Zbl 1093.55004 MR 2206643

[LöhS09] Clara Löh and Roman Sauer, Degree theorems and Lipschitz simplicial volume for non-positively curved manifolds of finite volume. J. Topol. 2 (2009), 193-225.

[MM85] Shigenori Matsumoto and Shigeyuki Morita, Bounded cohomology of certain groups of homeomorphisms. Proc. Amer. Math. Soc. 94 (3) (1985), 539-544.Zbl 0536.57023 MR 0787909

[Sto06] Peter A. Storm, The minimal entropy conjecture for nonuniform rank one lattices. Geom. Funct. Anal. 16 (4) (2006), 959-980. Zbl 1099.53041 MR 2255387

Received July 9, 2007

Clara Löh, Mathematisches Institut, Westfälische Wilhelms-Universität Münster,

Einsteinstr. 62, 48149 Münster, Germany

E-mail: clara.loeh@uni-muenster.de; www.math.uni-muenster.de/u/clara.loeh

Roman Sauer, Mathematisches Institut, Westfälische Wilhelms-Universität Münster, Einsteinstr. 62, 48149 Münster, Germany

E-mail: sauerr@uni-muenster.de; www.romansauer.de 